\title{
UV irradiation grafting of acrylamide onto dopamine-modified 316L stainless steel
}

\author{
Nan Yao $₫$, Jie Chen, Guochen Zhao, Ye Huang, Liming Yang, Hongbin Li, Zhenmei Sheng
}

(C) The Author(s) 2018

\begin{abstract}
UV irradiation grafting technique was applied on a modified 316L stainless steel (SS) surface. The SS sheets were first coated with dopamine by dipping in the dopamine solution with $\mathrm{KIO}_{3}$ and ultrasonication. Then, the acrylamide (AAm) was grafted onto the dopamine-modified SS samples under UV irradiation with ultrasonication, when the ketonediol compound was used as a photoinitiating system. For comparison, the UV irradiation grafting of AAm was also performed without ultrasonication. The structures and morphologies of the samples were examined by Fourier transform IR spectroscopy, atomic force microscopy, and scanning electronic microscopy before and after modification, respectively. Results showed that acrylamide was successfully grafted onto the poly-dopamine-coated SS. The hydrophilicity of the modified samples improved significantly, determined by the water contact angle measurement. Shown by Tafel polarization curves, the corrosion potential of modified SS was positively shifted, which demonstrated that the corrosion resistance was improved.
\end{abstract}

Keywords Acrylamide, Dopamine, 316L stainless steel, UV irradiation, Ultrasonication

N. Yao, J. Chen ( $\varangle)$, Y. Huang, L. Yang,

H. Li, Z. Sheng

School of Environmental and Chemical Engineering,

Shanghai University, Shanghai 200444, China

e-mail: jchen@shu.edu.cn

\section{G. Zhao}

Shangdong Key Laboratory for High Strength Lightweight Metallic Materials, Jinan 250000, China

\section{Introduction}

Surface modification strategies on biomedical stainless steel, such as polymer coating, plasma polymerization, and layer-by-layer self-assembly techniques, have been developed in recent years. ${ }^{1-3}$ Among the different surface modification means, the poly-dopamine (PDA) coating method has attracted great interest due to its good applicability, hydrophilicity, permeability, and stability to almost any substrate. ${ }^{4}$ Lee et al. ${ }^{5}$ reported that dopamine (3,4-dihydroxyphenethylamine) (DA) had the capacity to create strong coordinate bonds with various materials including metals to form a dense poly-dopamine film through a simple dip-coating method in an alkaline buffer solution. It is considered that poly-dopamine coating can be a versatile platform of secondary reactions for further diverse functional applications, which makes it feasible to improve the properties of materials in the biomedical fields. ${ }^{6,7}$ Therefore, it could be a proper pretreatment and mild condition for grafting functional polymers onto metallic materials.

It has been reported that photochemical initiation has several advantages, including fast initiation rate, low toxicity, as well as mild reaction conditions, which are very attractive for biologically relevant applications. ${ }^{8-10}$ Xin et al. ${ }^{11}$ found that dopamine polymerization can be induced or stopped by using UV light as a trigger, which makes it possible to form precise surface micropatterns of poly-dopamine and prepared for secondary reactions. Rahimpour et al. ${ }^{12}$ grafted acrylic acid onto the surface of PVDF membrane by UV irradiation grafting polymerization with benzophenone as photoinitiator. The hydrophilic and antifouling properties of the PVDF membranes improved significantly. Helin et al. ${ }^{13}$ synthesized the copolymers of polysulfone-graft-methyl acrylate by photoinitiated graft copolymerization. The methyl acrylate groups were presented on the copolymer membranes and 


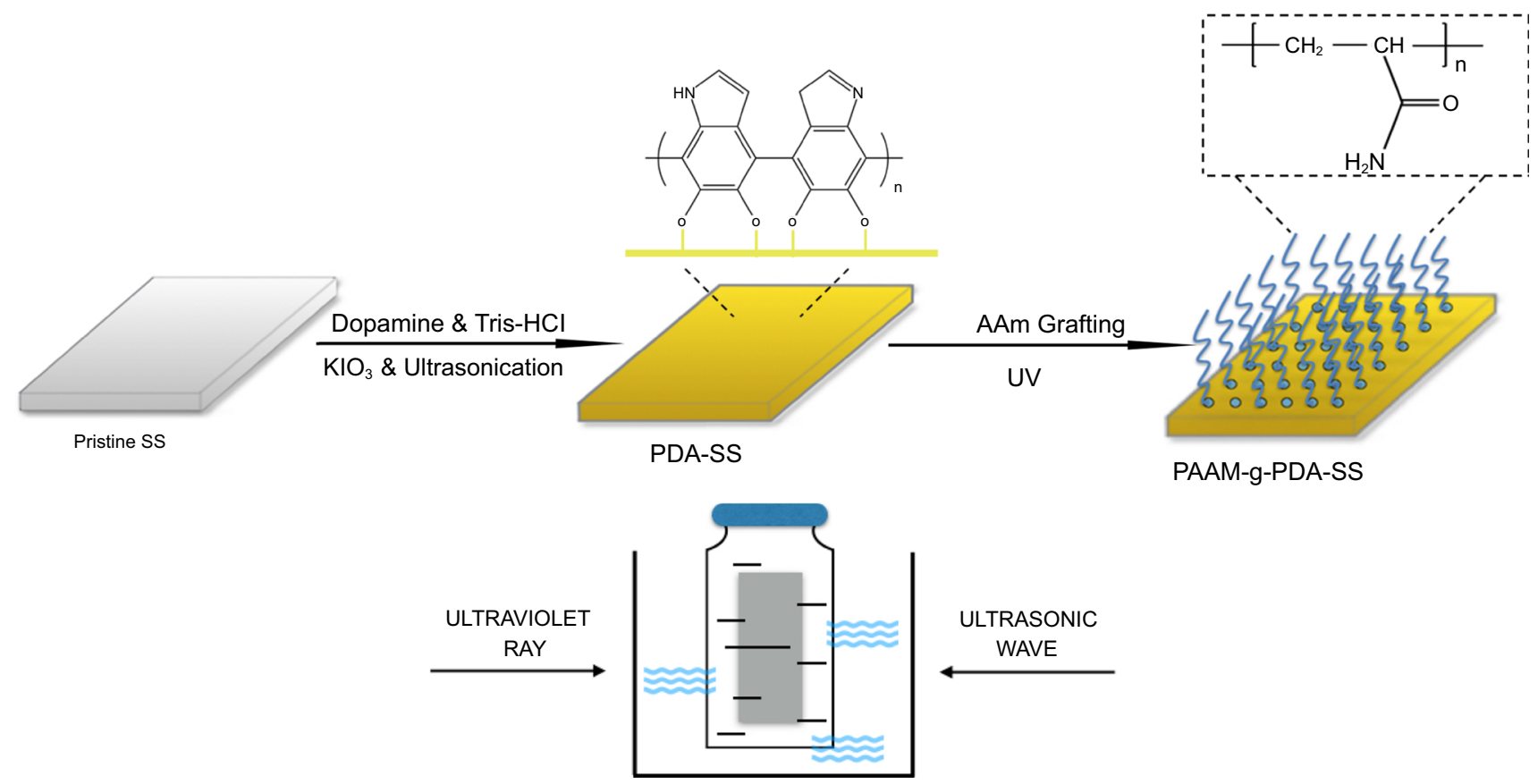

Scheme 1: Schematic representation of grafting AAm onto poly-dopamine-coated materials

greatly enhanced the antifouling property. Polyacrylamide, a macromolecule which can be synthesized by ultraviolet light and chemical initiator, is widely used in the biomedical field, such as for plastic surgery, drug release, and tissue engineering. ${ }^{14-16}$ Inspired by the application of acrylamide in the bone tissue engineering technique, a durable hydrophilic polymeric film could be formed on the surface of metallic materials by UV irradiation.

In this paper, 316L stainless steel, one of the most frequently used biomedical materials in medical and dental fields, ${ }^{17-19}$ was modified by dopamine with ultrasonication. $\mathrm{KIO}_{3}$ was also used as a strong oxidant to accelerate the formation time of poly-dopamine, ${ }^{20}$ which enabled it to be accomplished within $1 \mathrm{~h}$ as well as with better utilization of dopamine. Then, under the assistance of ultraviolet light irradiation, acrylamide was grafted onto the surfaces of poly-dopamine-coated samples in order to further improve the hydrophilicity of the surface, as illustrated in Scheme 1. The schematic representation of the formation process is shown in Scheme 2. Instead of the traditional benzophenone initiator, ${ }^{21}$ 2-butanone and propylene glycol- $(1,3)$ compound was employed as a low-toxicity photoinitiating system for the polymerization of acrylamide (AAm) to polyacrylamide (PAAm). After absorbing the photon generated from UV irradiation, either split by Norrish II reaction, the ketone molecules were stimulated and transitioned to the triplet state, and the dehydrogenation of alcohol molecules was able to generate free radicals and become attached onto the poly-dopamine coating, which could lead to grafting of acrylamide. The fate of most of the radicals is probably recombination to form nonreactive products. What is really

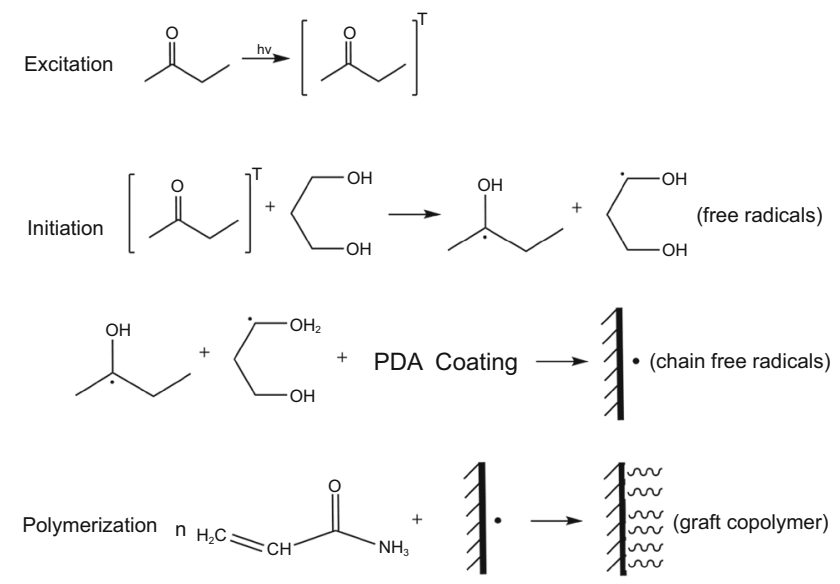

Scheme 2: Mechanism of grafting of AAm under ultraviolet light irradiation

important for the initiation of radicals is the nature of the excited states produced under UV irradiation. The excited states must have energies high enough to abstract hydrogen atoms and also have lifetimes long enough for the hydrogen abstraction to take place. Compared to the benzophenone photoinitiator, the ketone-diol compound is water soluble with multiple different photoactive groups, which may cause a cooperative effect at a low concentration. Energy transfer is limited by "diffusion control." The addition of ultrasonication was also able to cause a momentarily high temperature, and then, the linkages between water units were broken and free radicals with high activity were obtained in a short time. It may be helpful in the process of reactions at relatively lower activation 
energy and increase the free radical attachment to the coating. The reaction variables (i.e., concentrations of AAm, time and temperature) of radiation grafting process were investigated, and the ultrasonication effect to the samples was also compared in our research.

\section{Experimental}

\section{Instruments and materials}

The UV irradiation device was purchased from Shanghai Sineo Microwave Chemistry Technology Co., Ltd., China. The output power of the UV light source was $300 \mathrm{~W}$ and the center wavelength was $365 \mathrm{~nm}$. A threeelectrode configuration method consisting of the sample as working electrode, a platinum rod as counter electrode and a saturated calomel electrode as reference electrode were used. The working electrode area was set as $1 \mathrm{~cm}^{2}$. Potential of working electrodes was swept at a rate of $1 \mathrm{mV} \mathrm{s}^{-1}$ within the range of -1000 to $1000 \mathrm{mV}$ vs OCP. Dopamine- $\mathrm{HCl}$ was procured from Sigma-Aldrich, USA. Tris (hydroxymethyl aminomethane) was purchased from Sinopharm Chemical Reagent Co., Ltd., China. AAm was procured from Shanghai Green Analysis of Chemical Technology Co., Ltd., China. Acetone, 2-butanone and propylene glycol-(1,3) were received from Yixing Second Chemical Reagent, China. High purity nitrogen was from Shanghai Beor Gas Industry Co., Ltd., China. $\mathrm{KIO}_{3}$ and EDTA were purchased from Sinopharm Chemical Reagent Co., Ltd. All chemicals were analytically pure.

\section{Stainless steel surface process}

Undefiled stainless steel samples were polished in advance and cleaned with acetone and water under ultrasonic conditions for $15 \mathrm{~min}$. All the sheets were dried with nitrogen gas and cut into $1 \mathrm{~cm} \times 2 \mathrm{~cm}$ size before being used. The samples were labeled as SS.

\section{Deposition of poly-dopamine onto stainless steel}

The aqueous solution of dopamine $(0.04 \mathrm{M})$ which would be used for coating was prepared in the Tris$\mathrm{HCl}$ buffer solution of $\mathrm{pH} 9.5$ with $\mathrm{KIO}_{3}$ (a molar $\mathrm{KIO}_{3}$ /dopamine ratio of 2) to accelerate the formation time of poly-dopamine. The reaction happened at $30^{\circ} \mathrm{C}$ by immersing the undefiled stainless steel sample into the solution with the help of ultrasonication for $1 \mathrm{~h}$ in the air. Ultrasonic frequency was $26-28 \mathrm{kHz}$. To eliminate useless chemicals, the coated samples were rinsed with a large amount of ultra-pure water and dried under a flowing nitrogen environment for further experiments. For comparison, the deposition of polydopamine was also performed without ultrasonication. The samples were labeled as PDA-SS.

\section{UV grafting of AAm onto the surface of PDA-SS}

The dopamine-coated stainless steel sheet (PDA-SS obtained with ultrasonication) was immersed in a reaction mixture of acrylamide (AAm) aqueous solution, which was composed of acrylamide, 2-butanone and propylene glycol- $(1,3)$ compound (mass ratio 1:1, $2 \mathrm{~mL} / \mathrm{L})$, EDTA $(0.04 \mathrm{wt} \%)$ and ultra-pure water in a quartz ampule $(5 \mathrm{~mL})$. Nitrogen was bubbled in the reaction solution for $15 \mathrm{~min}$ to exclude oxygen completely. The bottle was settled in a thermostatic quartz water tank. The grafting reaction took place by irradiating the reaction ampule with ultraviolet (UV) light under ultrasonication, and each sample was put at a uniform distance of $25 \mathrm{~cm}$ close to the center of UV light in order to get the same illumination intensity. For comparison, the UV irradiation grafting reaction was also performed without ultrasonication. After irradiation grafting, the sheet was washed by immersing into ultra-pure water and shaking for over $24 \mathrm{~h}$. Water was changed every $2 \mathrm{~h}$. The samples were labeled as PAAm-g-PDA-SS.

\section{Surface characterization}

The surface chemical structures of bare SS, PDA-SS and PAAm-g-PDA-SS samples were verified by a Fourier transform IR (FTIR) spectrophotometer in the attenuated total reflectance mode. The surface morphologies of samples were observed by an atomic force microscope (AFM), (Dimension Icom, Bruker, USA) and scanning electron microscope (SEM), (EVO MA10, ZEISS, GER). The hydrophilicity of the samples was studied by measuring the water contact angles using a water contact angle meter (OCA20, Dataphysics Instruments $\mathrm{GmbH}, \mathrm{GER})$ at $25^{\circ} \mathrm{C}$, relative humidity $60 \%$. Tafel polarization curves were drawn to estimate the corrosion resistance of samples by the electrochemical workstation (GillAC ACM, UK).

\section{Results and discussion}

Both poly-dopamine coating and AAm-grafted SS samples were obtained under the static condition and ultrasonication, respectively. The effects of reaction conditions, such as ultrasonication, reaction temperature, reaction time and AAm concentration on the morphology, water contact angle and corrosion resistance of the SS sample were studied. 
(a)
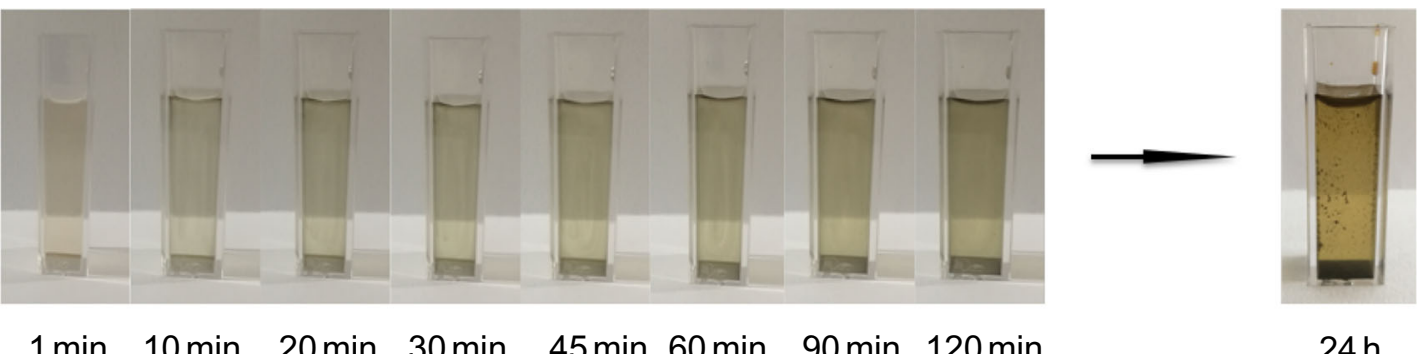

$24 \mathrm{~h}$

(b)

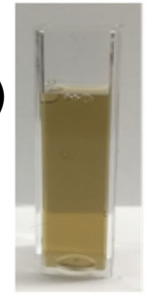

$1 \min$

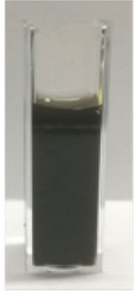

$10 \mathrm{~min}$

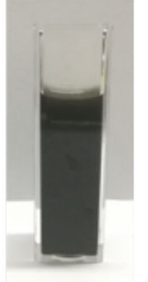

$30 \mathrm{~min}$

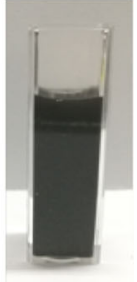

$60 \mathrm{~min}$

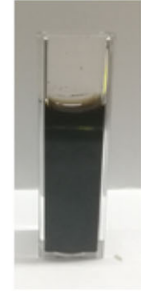

$24 \mathrm{~h}$

Fig. 1: Color changes of dopamine solutions. (a) Static solutions at pH 9.5 without $\mathrm{KIO}_{3}$ and ultrasonication, (b) $\mathrm{KIO}_{3}$ /dopamine solutions at $\mathrm{pH} 9.5$ with ultrasonication

\section{Deposition of dopamine}

The ultrasonication deposition of dopamine was finished in $1 \mathrm{~h}$, which was shorter than that of static deposition. The poly-dopamine deposited with ultrasonication was steadily anchored onto the surface of SS, while the counterpart could be brushed off easily with filter paper, when the deposition process was performed in the static situation. The deposition rate of dopamine could also be observed from the color changes of dopamine solutions in different situations. Deep dark solution and precipitation of large PDA particles visible with the naked eye came to exist after $24 \mathrm{~h}$. The basic solutions at $\mathrm{pH} 9.5$ exhibited a slow color change tendency when there was no additional auxiliary means. The process of the dopamine deposition happened in a short time with ultrasonication in the $\mathrm{KIO}_{3}$ /dopamine solution, which can be clearly seen in Fig. 1.

\section{Fourier transform IR (FTIR) spectroscopy}

Samples of PDA-SS obtained in the coating condition of ultrasonication and PAAm-g-PDA-SS obtained in the reaction condition of 120-min UV irradiation with ultrasonication in $30 \%$ AAm aqueous solution at $30^{\circ} \mathrm{C}$ were studied using the FTIR spectrometer with the background of plain stainless steel. As shown in Fig. 2, a broad absorbance between 3600 and $3100 \mathrm{~cm}^{-1}$ is ascribed to $\mathrm{N}-\mathrm{H} / \mathrm{O}-\mathrm{H}$ stretching vibrations of the polydopamine coating. Peaks at 1615 and $1502 \mathrm{~cm}^{-1}$ are

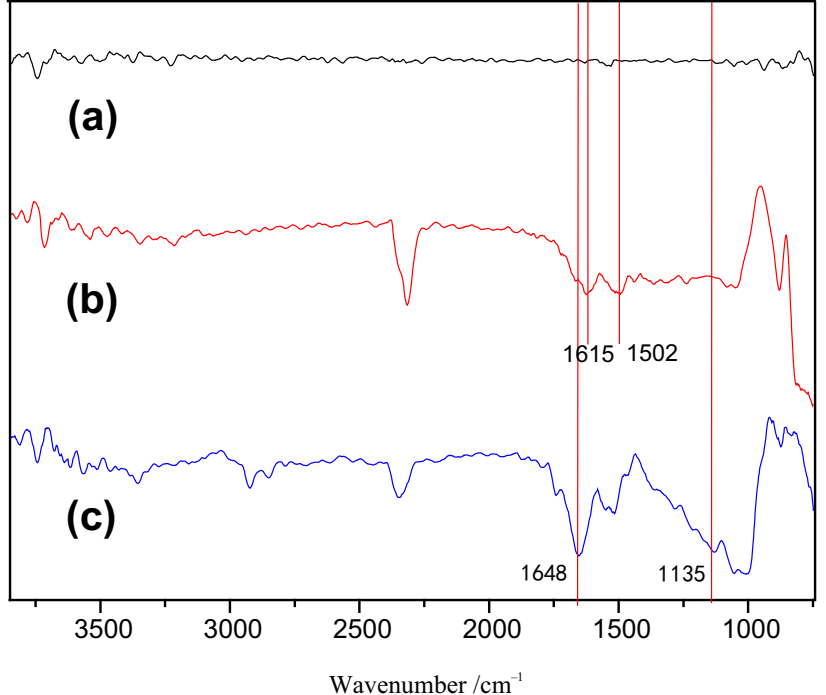

Fig. 2: IR spectra of samples. (a) SS, (b) PDA-SS and (c) PAAm-g-PDA-SS

attributed to the overlap of the $\mathrm{C}=\mathrm{C}$ resonance vibrations in the aromatic ring and $\mathrm{N}-\mathrm{H}$ bending vibrations, ${ }^{22}$ respectively, which means the bare stainless steel has been covered with poly-dopamine. Compared to the PDA-SS, new absorption bands at 1648 and $1135 \mathrm{~cm}^{-1}$ in the spectrum of PAAm-g-PDASS correspond to the $\mathrm{C}=\mathrm{O}$ and $\mathrm{C}-\mathrm{N}$ of polyacrylamide. ${ }^{23}$ These changes of characteristic peaks indicated that AAm was successfully grafted onto the surface of the PDA-SS sheet. 


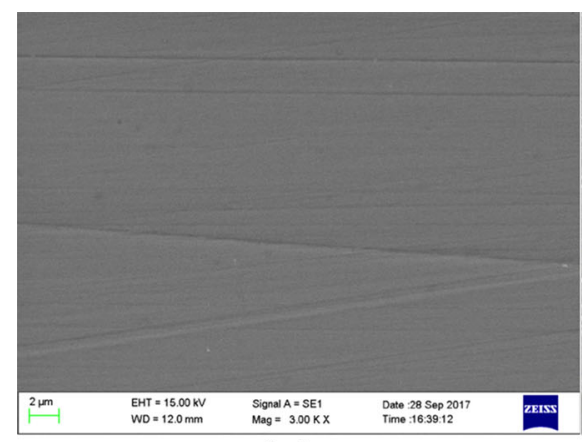

(a)

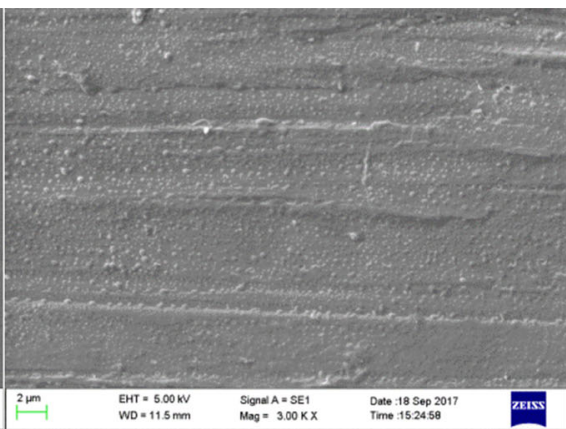

(b)

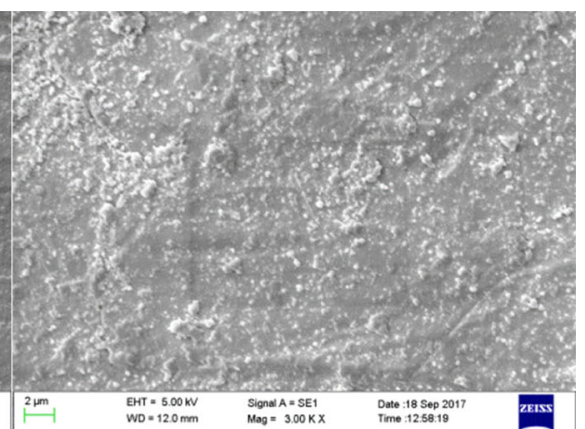

(c)

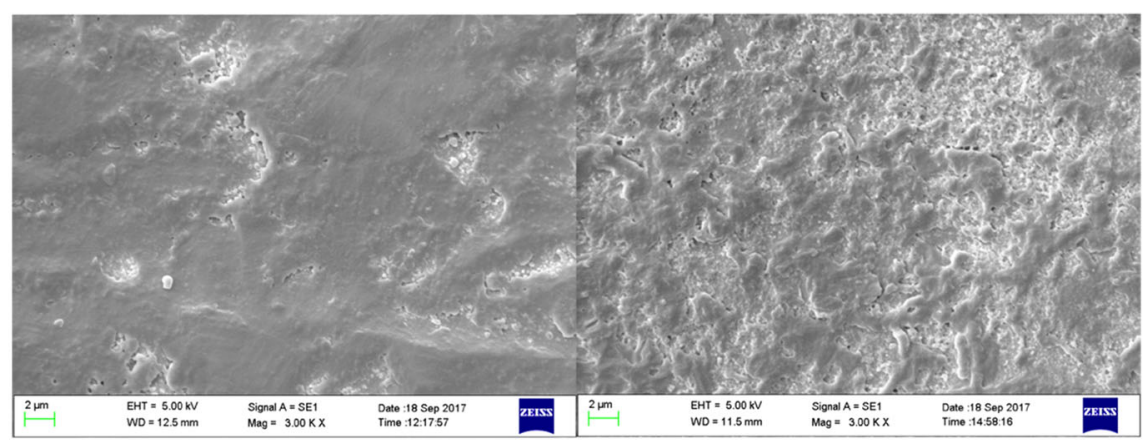

(d)

(e)

Fig. 3: SEM images of samples. (a) SS, (b) PDA-SS with ultrasonication, (c) PAAm-g-PDA-SS with ultrasonication, (d) PDASS in static situation, and (e) PAAm-g-PDA-SS in static situation

\section{Surface morphologies of samples}

\section{Scanning electron microscopy (SEM)}

The surface morphologies of SS, PDA-SS and PAAmg-PDA-SS sheets were observed using a scanning electron microscope. As shown in Fig. 3, the surface of the PDA-SS (Fig. 3b, obtained in the $\mathrm{KIO}_{3} /$ dopamine solution with ultrasonication) became rougher than the bare stainless steel (Fig. 3a). Besides, the particles were relatively larger after being grafted with AAm (Fig. 3c, obtained in the condition of $30^{\circ} \mathrm{C}, 30 \%$ AAm, 120 min under UV irradiation with ultrasonication). Hence, it suggested that AAm was successfully grafted onto the surface of PDA-SS. Compared with samples obtained in static situation (Figs. 3d, 3e), the difference of smoothness and thickness of the coating can be distinctly seen due to the residuary homopolymer on the samples. It is suggested that the surface appearance can be further smoothened by ultrasonication.

\section{Atomic force microscopy (AFM)}

The AFM results conformed to aforementioned inference. It is shown that ultrasonication has an effect on the surface thickness of samples in Fig. 4. The average thickness values of samples, as listed in Table 1, tended to augment when the samples were obtained in static situation. Results showed the average thickness value of PDA-SS (Fig. 4a, obtained in the $\mathrm{KIO}_{3}$ /dopamine solution with ultrasonication) was $802 \mathrm{~nm}$, much lower than $940 \mathrm{~nm}$ when there was no ultrasonication (Fig. 4c). The average thickness values of PDA-SS measured 1778 and $1965 \mathrm{~nm}$ after being grafted with AAm in ultrasonication and static situation, respectively (Figs. 4b, 4d). The distinct changes in surface morphology after modification further confirmed the presence of polymer coating on the surface of stainless steel.

\section{Water contact angle test}

The surface differences of samples were also demonstrated by the changes in water contact angles as shown in Fig. 5. The water contact angle of bare stainless steel was $71.2^{\circ}$, while it was $52.1^{\circ}$ and $33.5^{\circ}$ for PDA-SS and PAAm-g-PDA-SS (obtained in static condition of $30^{\circ} \mathrm{C}, 30 \%$ AAm, $120 \mathrm{~min}$ in all). This suggested that the surface of stainless steel became hydrophilic after coating with dopamine and became futher hydrophilic after AAm grafting onto the surface. Results showed the water contact angles can be further decreased to $49.7^{\circ}$ and $24.5^{\circ}$ for PDA-SS and PAAm-g-PDA-SS with ultrasonication. The effects of grafting conditions on the surface hydrophilic property of PDA-SS sheets 


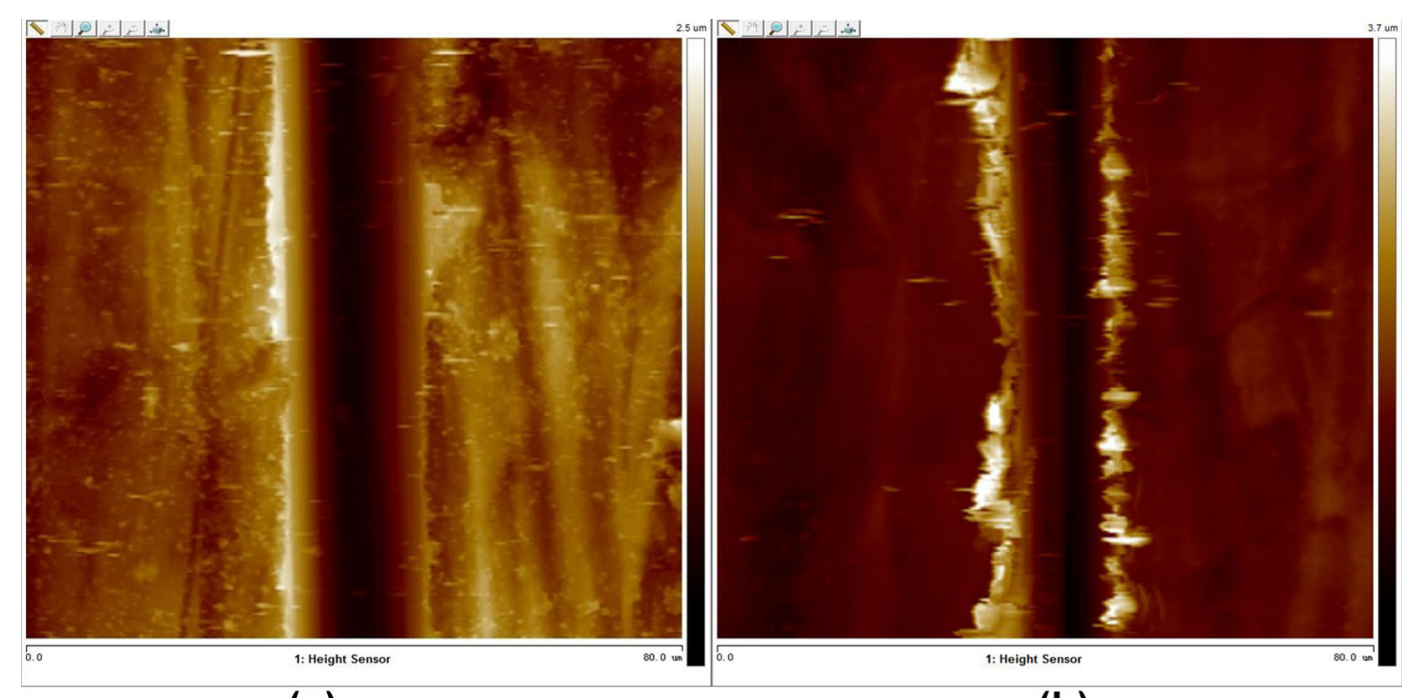

(a)

(b)

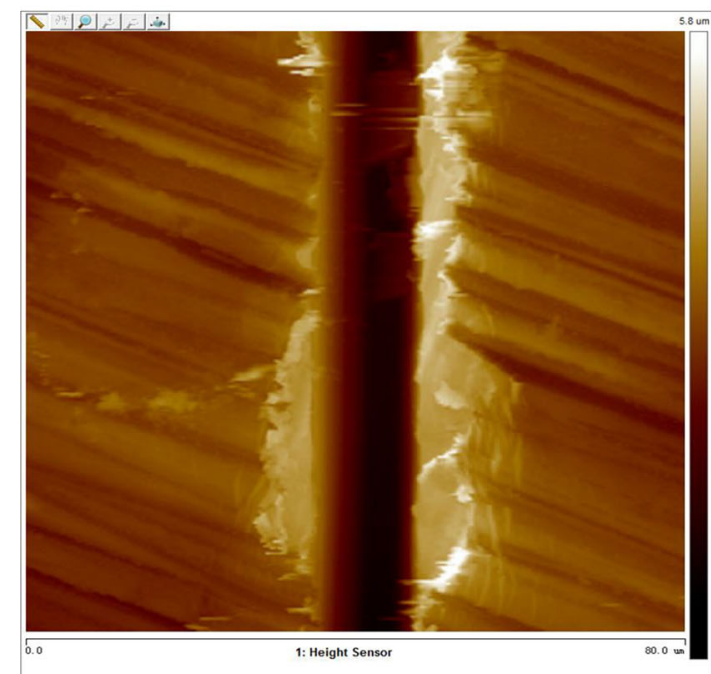

(c)

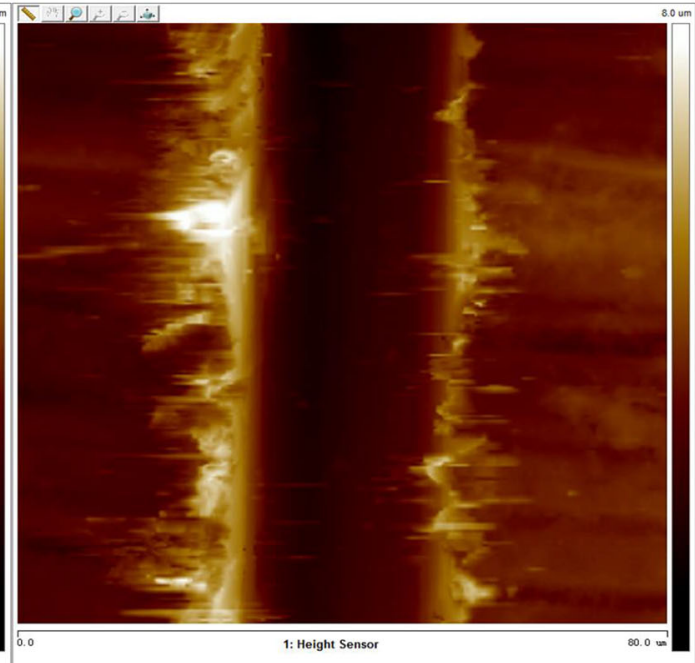

(d)

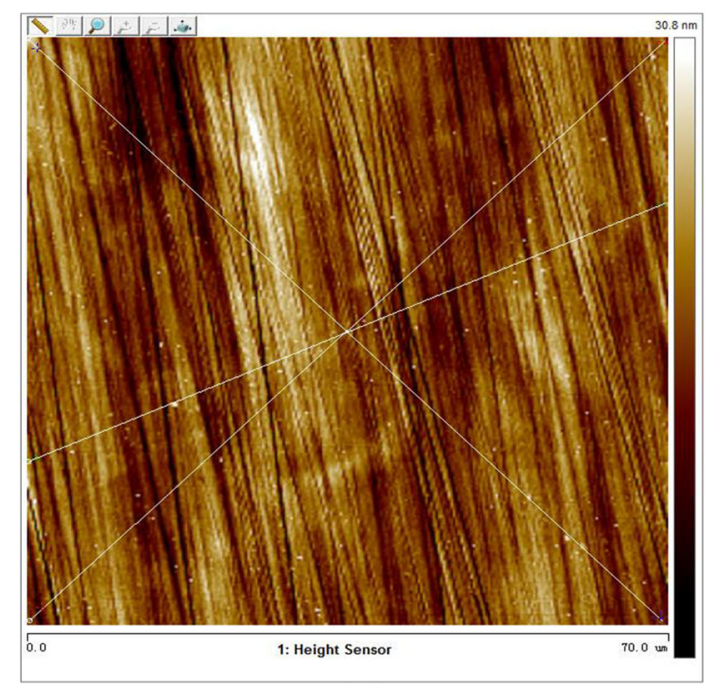

(e) 
4Fig. 4: AFM images of samples. (a) PDA-SS with ultrasonication, (b) PAAm-g-PDA-SS with ultrasonication, (c) PDA-SS in static situation, (d) PAAm-g-PDA-SS in static situation, and (e) SS

Table 1: The average thickness values of different samples

\begin{tabular}{lcccc} 
Samples & a & b & c & d \\
\hline Average thickness/nm & 802 & 1778 & 940 & 1965
\end{tabular}

(a) PDA-SS with ultrasonication, (b) PAAm-g-PDA-SS with ultrasonication, (c) PDA-SS in static situation, (d) PAAm-gPDA-SS in static situation

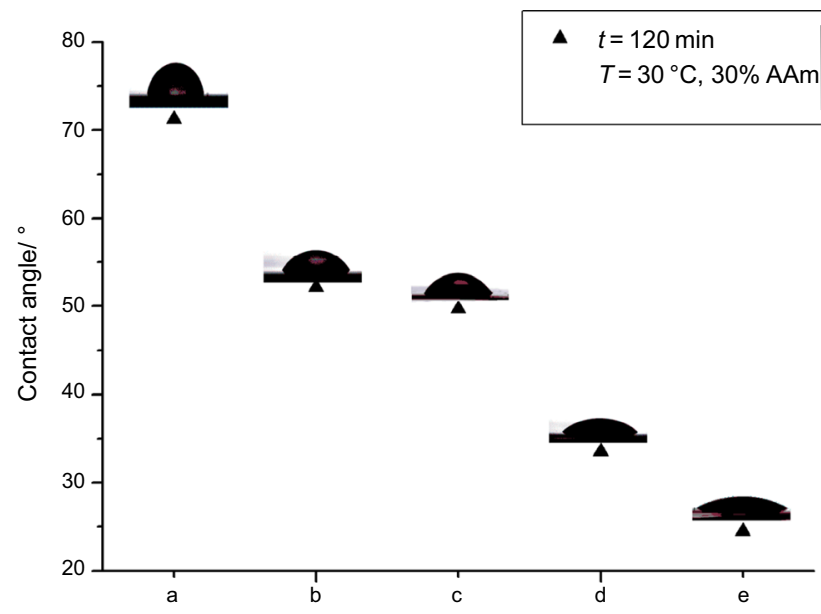

Fig. 5: Water contact angle of samples. (a) SS, (b) PDA-SS in static situation, (c) PDA-SS with ultrasonication, (d) PAAm-g-PDA-SS in static situation, and (e) PAAm-g-PDASS with ultrasonication

were studied by changing the concentrations of AAm, reaction time, and reaction temperature, respectively.

\section{Reaction temperature}

The effects of temperature on the water contact angles of samples are demonstrated in Fig. 6 (obtained in the condition of $30 \%$ AAm, $120 \mathrm{~min}$ in all). As the temperature rose from 30 to $50^{\circ} \mathrm{C}$, the contact angle grew gradually from $33.1^{\circ}$ to $43.5^{\circ}$. This is mainly because of the increasing active chain termination rate caused at higher temperature. The free radicals initiated at first had difficulty surviving with the rising reaction heat. ${ }^{24}$ The contact angles' change of samples in both ultrasonic and ordinary conditions was compared. Each sample indicated a decline of contact angle with the addition of ultrasonication, which could even drop to $24.5^{\circ}$ at $30^{\circ} \mathrm{C}$. The results showed that the ultrasonication and lower temperature were beneficial to improving the hydrophilicity of the samples.

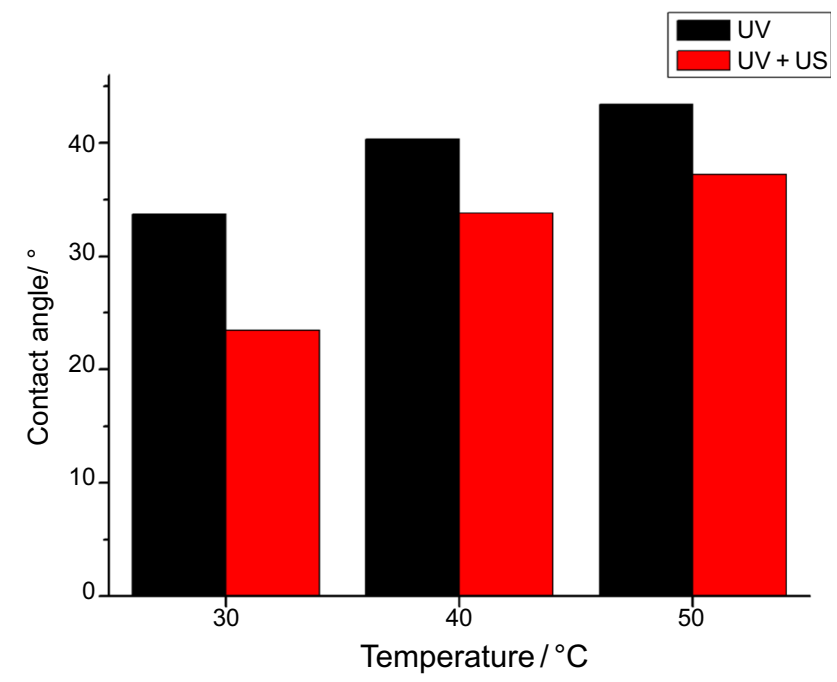

Fig. 6: Effect of temperature on water contact angle in comparison with static (UV) and ultrasonication (UV + US) conditions (reaction condition: $30 \% \mathrm{AAm}, 120 \mathrm{~min}$ in all)

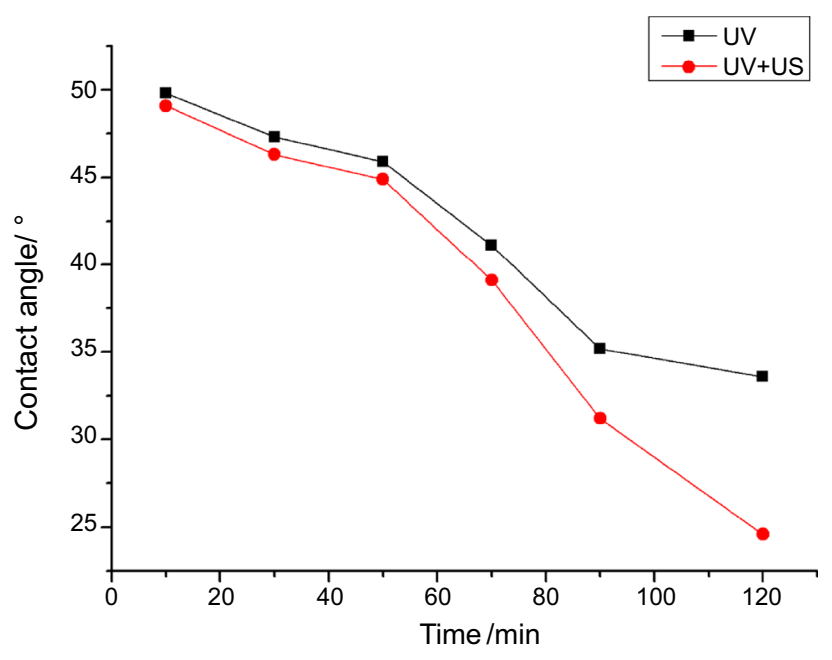

Fig. 7: Effect of illumination time on water contact angle in comparison with static (UV) and ultrasonication (UV + US) conditions (reaction condition: $30^{\circ} \mathrm{C}, 30 \% \mathrm{AAm}$ )

\section{Reaction time}

Figure 7 illustrates the change of contact angles when the reaction time increased gradually in a fixed condition (obtained in the condition of $30^{\circ} \mathrm{C}, 30 \%$ AAm). The water contact angle decreased slowly at the first $50 \mathrm{~min}$, from $49.7^{\circ}$ to $45.9^{\circ}$, and eventually fell to $33.5^{\circ}$ in a static situation when it reached $120 \mathrm{~min}$. It has been shown that the water contact angle could be further reduced to $24.5^{\circ}$ with ultrasonication. With time passing, it is suggested that the gel effect would occur and the accumulated polymeric heat could restrict further grafting reaction. Furthermore, it had reached the top hydrophilicity of acrylamide. Therefore, $120 \mathrm{~min}$ was selected as the optimal reaction time. 


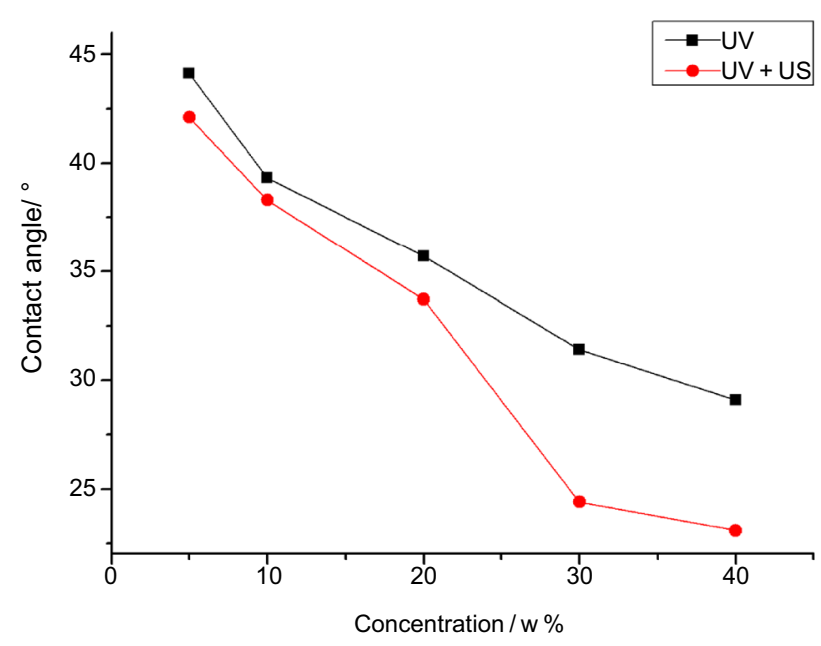

Fig. 8: Effect of AAm concentration on water contact angle in comparison with static (UV) and ultrasonication (UV + US) conditions (reaction condition: $30^{\circ} \mathrm{C}, 120 \mathrm{~min}$ in all)

\section{AAm concentration}

Figure 8 shows the dependence of water contact angle on the AAm concentration when other parameters were fixed (obtained in the condition of $30^{\circ} \mathrm{C}, 120 \mathrm{~min}$ in all). It showed that water contact angles decreased greatly with the AAm concentration up from $5 \%$ to $30 \%$, reaching from $42.2^{\circ}$ to $24.5^{\circ}$ with ultrasonication. When the AAm concentration varied to $40 \%$, free radicals were hard to diffuse in a short time due to the high viscosity of the system. The ultrasonication could hardly further improve the grafting effect at such high density of solution. There was no significant change of the water contact angle but the cost of AAm increased about one-third. Thus, $30 \%$ was assumed to be the optimal concentration.

\section{Corrosion resistance test}

In order to compare the corrosion resistance before and after modification, Tafel plots were carried out in the $\mathrm{NaCl}$ solution $(3.0 \%)$ at $25^{\circ} \mathrm{C}$, as shown in Fig. 9 . The corrosion potential of bare SS was $-355 \mathrm{mV}$, and after modification, the corrosion potential of PDA-SS and PAAm-g-PDA-SS shifted to -274 and $-231 \mathrm{mV}$ (both obtained in the condition of $30^{\circ} \mathrm{C}, 30 \%$ AAm, $120 \mathrm{~min}$ in all), which meant that the corrosion resistance of SS after modification was better than that of bare SS. Therefore, the PAAm coating can help to improve the corrosion potential of SS in the neutral solution.

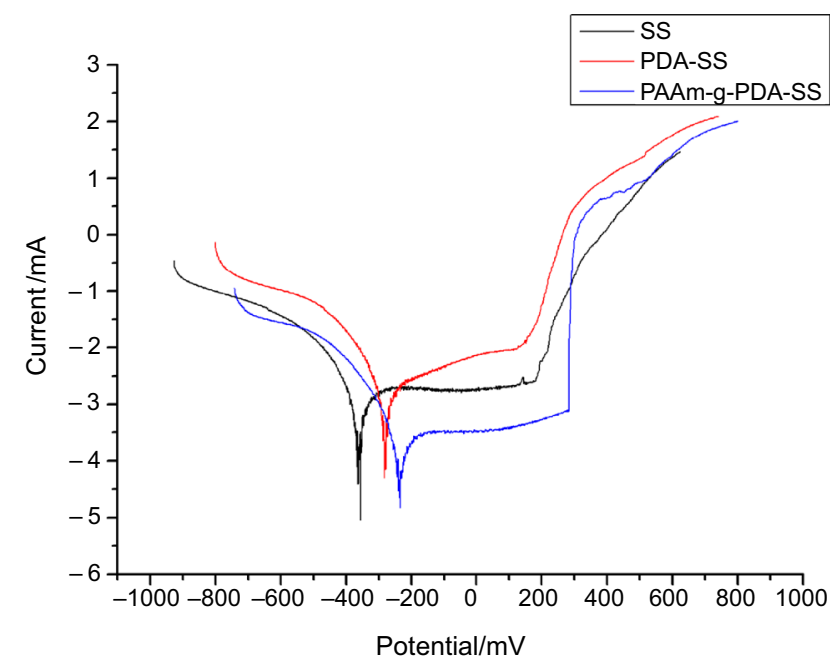

Fig. 9: Tafel polarization curve of SS, PDA-SS and PAAmg-PDA-SS, in $\mathrm{NaCl}$ solution (3.0\%) at $25^{\circ} \mathrm{C}$

\section{Conclusions}

Acrylamide was successfully grafted onto the surface of poly-dopamine-coated stainless steel by UV irradiation, which can be concluded from the SEM images and FTIR spectra. The surface of medical 316L stainless steel was modified by dopamine coating with ultrasonication. Then the acrylamide was grafted onto the dopamine-modified samples under UV irradiation, when the ketone-diol compound was used as a photoinitiating system. It was shown that the corrosion resistance and the hydrophilicity of the stainless steel surface were obviously improved after the coating of dopamine and the further grafting of acrylamide. The ultrasonic condition could also help to improve the grafting effect. To sum up, ultrasonic UV irradiation grafting is an effective approach for preparing durable hydrophilic metallic surfaces. We believe that the materials mentioned above have great potential for applications in the medical and dental fields.

Acknowledgment The authors would like to express their thanks to financial supports from the National Key Research and Development Plan (Grant No. 2017YFB0103904).

Open Access This article is distributed under the terms of the Creative Commons Attribution 4.0 International License (http://creativecommons.org/lice nses/by/4.0/), which permits unrestricted use, distribution, and reproduction in any medium, provided you give appropriate credit to the original author(s) and the source, provide a link to the Creative Commons license, and indicate if changes were made. 


\section{References}

1. Bertrand, P, Jonas, A, Laschewsky, A, et al., "Ultrathin Polymer Coatings by Complexation of Polyelectrolytes at Interfaces: Suitable Materials, Structure and Properties." Macromol. Rapid Commun., 21 (7) 319-348 (2015)

2. Finke, B, Luethen, F, Schroeder, K, et al., "The Effect of Positively Charged Plasma Polymerization on Initial Osteoblastic Focal Adhesion on Titanium Surfaces." Biomaterials, 28 (30) 4521-4534 (2007)

3. Sukhishvili, SA, Granick, S, "Layered, Erasable Polymer Multilayers Formed by Hydrogen-Bonded Sequential SelfAssembly." Macromolecules, 35 (1) 301-310 (2015)

4. Ryu, J, Ku, SH, Lee, H, et al., "Mussel-Inspired Polydopamine Coating as a Universal Route to Hydroxyapatite Crystallization." Adv. Funct. Mater., 20 (13) 2132-2139 (2010)

5. Lee, H, Dellatore, SM, Miller, WM, et al., "Mussel-Inspired Surface Chemistry for Multifunctional Coatings." Science, 318 (5849) 426 (2007)

6. Wei, Q, Zhang, F, Li, J, et al., "Oxidant-Induced Dopamine Polymerization for Multifunctional Coatings." Polym. Chem., 1 (9) 1430-1433 (2010)

7. Wang, JL, Li, BC, Li, ZJ, et al., "Electropolymerization of Dopamine for Surface Modification of Complex-Shaped Cardiovascular Stents." Biomaterials, 35 (27) 7679-7689 (2014)

8. Campos, LM, Killops, KL, Sakai, R, et al., "Development of Thermal and Photochemical Strategies for Thiol-Ene Click Polymer Functionalization." Macromolecules, 41 (19) 7063 7070 (2008)

9. Ichimura, K, "A Convenient Photochemical Method to Immobilize Enzymes." J. Polym. Sci. Part A Polym. Chem., 22 (11) 2817-2828 (2010)

10. Aluker, ED, Krechetov, AG, Mitrofanov, AY, et al., "Topography of Photochemical Initiation in Molecular Materials." Molecules, 18 (11) 14148-14160 (2013)

11. Du, X, Li, L, Li, J, et al., "UV-Triggered Dopamine Polymerization: Control of Polymerization, Surface Coating, and Photopatterning." Adv. Mater., 26 (47) 8029-8033 (2014)

12. Rahimpour, A, Madaeni, SS, Zereshki, S, et al., "Preparation and Characterization of Modified Nano-Porous PVDF Membrane with High Antifouling Property Using UV Photo-Grafting." Appl. Surf. Sci., 255 (16) 7455-7461 (2009)

13. Helin, H, Linlin, WU, Zhong, $\mathrm{H}$, et al., "Anti-Fouling Ultrafiltration Membrane Prepared from Polysulfone-Graft-
Methyl Acrylate Copolymers by UV-Induced Grafting Method." J. Environ. Sci., 20 (5) 565-570 (2008)

14. Yang, S, Hu, J, Chen, C, et al., "Mutual Effects of Pb(II) and Humic Acid Adsorption on Multiwalled Carbon Nanotubes/ Polyacrylamide Composites from Aqueous Solutions." Environ. Sci. Technol., 45 (8) 3621 (2011)

15. Ulbricht, M, "Advanced Functional Polymer Membranes." Polymer, 47 (7) 2217-2262 (2006)

16. Fang, X, Xie, J, Zhong, L, et al., "Biomimetic Gelatin Methacrylamide Hydrogel Scaffolds for Bone Tissue Engineering." J. Mater. Chem. B, 4 (6) 1070-1080 (2016)

17. Shen, GX, Chen, YC, Lin, CJ, "Corrosion Protection of 316L Stainless Steel by a $\mathrm{TiO}_{2}$, Nanoparticle Coating Prepared by Sol-Gel Method." Thin Solid Films, 489 (1-2) 130-136 (2005)

18. Dewidar, MM, Khalil, KA, Lim, JK, "Processing and Mechanical Properties of Porous 316L Stainless Steel for Biomedical Applications." Trans. Nonferrous Metals Soc. China, 17 (3) 468-473 (2007)

19. Yang, Z, Wang, J, Luo, R, et al., "The Covalent Immobilization of Heparin to pulsed-Plasma Polymeric Allylamine Films on 316L Stainless Steel and the Resulting Effects on Hemocompatibility." Biomaterials, 31 (8) 2072 (2010)

20. Bernsmann, F, Ball, V, Addiego, F, et al., "DopamineMelanin Film Deposition Depends on the used Oxidant and Buffer Solution." Langmuir ACS J. Surf. Colloids, 27 (6) 2819 (2011)

21. Leprince, JG, Hadis, M, Shortall, AC, et al., "Photoinitiator type and applicability of exposure reciprocity law in filled and unfilled photoactive resins." Dent. Mater., 27 (2) 157-164 (2011)

22. Cheng, C, Li, S, Zhao, W, et al., "The Hydrodynamic Permeability and Surface Property of Polyethersulfone Ultrafiltration Membranes with Mussel-Inspired Polydopamine Coatings." J. Membr. Sci., 417-418 (11) 228-236 (2012)

23. Yang, Z, Wang, J, Luo, R, et al., "The Covalent Immobilization of Heparin to Pulsed-Plasma Polymeric Acrylamide Films on 316L Stainless Steel and the Resulting Effects on Hemocompatibility." Biomaterials, 31 (8) 2072 (2010)

24. Galperin, A, Long, TJ, Ratner, BD, "Degradable, ThermoSensitive Poly(N-isopropyl acrylamide)-Based Scaffolds with Controlled Porosity for Tissue Engineering Applications." Biomacromolecules, 11 (10) 2583-2592 (2010) 